\title{
Conservação pós-colheita de alface cultivada com composto orgânico(1)
}

\author{
Ricardo Henrique Silva Santos ${ }^{(2)}$, Franceli da Silva(2), Vicente Wagner Dias Casali(2) \\ e Alcides Reis Condé(3)
}

\begin{abstract}
Resumo - O objetivo deste trabalho foi avaliar o efeito da adubação com composto orgânico, na presença e na ausência de adubação mineral padrão, na conservação pós-colheita das plantas de alface cultivar Babá. O composto orgânico foi aplicado nas doses de 0,0,22,8, 45,6,68,4 e 91,2 t/ha de matéria seca. Foram avaliadas a perda de matéria fresca em condições de ambiente e a degradação da clorofila em câmara fria $\left(4^{\circ} \mathrm{C}\right)$ até 84 horas e 12 dias, respectivamente, após colheita. A aplicação de doses crescentes de composto orgânico reduziu a perda de matéria fresca após a colheita em até 7\%; os teores de clorofila decresceram durante o armazenamento, em plantas adubadas com 45,6 e 91,2 t/ha de composto orgânico e adubo mineral, atingindo cerca de $0,3 \mathrm{mg} / \mathrm{g}$ de tecido fresco. A aplicação conjunta de adubo mineral e composto acelerou a senescência de alfaces mantidas em câmara fria, mas o cultivo com composto orgânico reduziu a perda de matéria fresca em condições de ambiente
\end{abstract}

Termos para indexação: Lactuca sativa, adubos compostos, adubos orgânicos, fisiologia pós-colheita, perdas, senescência.

\section{Post harvest conservation of lettuce cultivated with organic compost}

\begin{abstract}
This paper aimed to evaluate the effect of organic compost fertilization on the post harvest conservation of 'Babá' lettuce. The organic compost was applied at 0.0, 22.8, 45.6, 68.4 and $91.2 \mathrm{ton} / \mathrm{ha}$ (dry matter based), with and without a standard mineral fertilizer dosage. The fresh weight loss at room conditions and the chlorophyll degradation were evaluated in cold chamber $\left(4^{\circ} \mathrm{C}\right)$ until 84 hours and 12 days after harvest, respectively. Increasing rates of organic compost reduced the post harvest fresh weight loss up to $7 \%$. The chlorophyll content decreased during storage when plants were grown with 45.6 and 91.2 ton/ha of organic compost plus mineral fertilizer, reaching values of $0.3 \mathrm{mg} / \mathrm{g}$ of fresh matter. The fertilization with organic compost and mineral fertilizer altogether resulted in plants with early senescence when stored in cold chamber. Increasing doses of organic compost resulted in plants with smaller fresh weight loss in shelf conditions.
\end{abstract}

Index terms: Lactuca sativa, compound fertilizers, organic fertilizers, post harvest physiology, losses, senescence.

\section{Introdução}

A alface é a hortaliça folhosa mais consumida no Brasil; normalmente, é produzida em cinturões verdes próximos aos grandes centros consumidores por causa de sua rápida perecibilidade no período póscolheita, oriunda de seu alto teor de água e grande área foliar. $\mathrm{O}$ processo de senescência de folhosas

\footnotetext{
(1) Aceito para publicação em 16 de junho de 2000.

Trabalho financiado pela FAPEMIG.

(2)Universidade Federal de Viçosa (UFV), Dep. de Fitotecnia, CEP 36571-000 Viçosa, MG. E-mail: rsantos@mail.ufv.br, fsilva@bol.com.br, vwcasali@mail.ufv.br

(3)UFV, Dep. de Matemática. E-mail: dpi@ufv.br
}

ocorre principalmente em virtude do déficit hídrico proveniente da rápida perda de água (Wills et al., 1981) e da ação de diversas enzimas catalíticas. A redução da temperatura aumenta a conservação pós-colheita de folhosas, por diminuir a diferença de pressão de vapor entre a planta e o meio, reduzindo a perda de água (Wills et al., 1981). A colheita interrompe o suprimento de água para o órgão vegetal, e assim, a perda de água subseqüente por transpiração determinará, em grande parte, as perdas quantitativas e qualitativas dos produtos. A perda de água pode acelerar a deterioração, pelo aumento da taxa de algumas reações de origem predominantemente catabólica, como a degradação da clorofila (Finger \& Vieira, 1997). O grau de senescência da alface é 
eficientemente indicado pela degradação da clorofila (Silva, 1980; Medina et al., 1982).

A adoção da adubação orgânica no cultivo de hortaliças tem crescido nos últimos anos, devido, principalmente, aos efeitos benéficos do material orgânico sobre as características físicas e químicas do solo, pelo custo elevado dos adubos minerais solúveis e ao marketing realizado em torno da produção orgânica de alimentos. Aumentos de produtividade de alface em razão do aumento de doses de matéria orgânica são relatados por Hoffman \& Schweiger (1983) e Hernandez et al. (1992), citados por Santos et al. (1998). A adubação orgânica não só incrementa a produtividade mas também proporciona a obtenção de plantas com características qualitativas distintas das cultivadas exclusivamente com adubos minerais (Santos et al., 1994), podendo, portanto, exercer influência sobre a conservação pós-colheita da alface.

O objetivo deste trabalho foi verificar o efeito da adubação com composto orgânico, na presença e ausência de adubo mineral, na conservação pós-colheita de plantas de alface, cultivar Babá.

\section{Material e Métodos}

O experimento foi realizado na horta da Universidade Federal de Viçosa (UFV), em Viçosa, MG, estabelecendose dez tratamentos, constituídos de cinco doses de composto orgânico (ORG) na presença (1) e na ausência (0) de adubo mineral (MIN), arranjados em fatorial 5 x 2, em blocos casualizados, com quatro repetições

As doses de composto orgânico foram de $0,0,22,8$, 45,6, 68,4 e 91,2 t/ha de matéria seca, e a adubação mineral seguiu recomendação da Comissão de Fertilidade do Solo do Estado de Minas Gerais (1989), constando de $643 \mathrm{~kg} / \mathrm{ha}$ da fórmula 4-14-8 ou seja, 25,7, 90,0 e $51,4 \mathrm{~kg} /$ ha de $\mathrm{N}, \mathrm{P}_{2} \mathrm{O}_{5}$ e $\mathrm{K}_{2} \mathrm{O}$ respectivamente, aplicados na época do transplante das mudas, e duas adubações de cobertura de $30 \mathrm{~kg} / \mathrm{ha}$ de $\mathrm{N}$ cada, aplicadas 15 e 37 dias após o transplante. O composto orgânico apresentou $17,38 \%$ de (C) orgânico, $1,92 \%$ de N-total, $1,30 \%$ de P-total, $1,58 \%$ de $\mathrm{K}, 2,48 \%$ de $\mathrm{Ca}, 0,42 \%$ de $\mathrm{Mg}$, e relação C:N de 9:1, determinados segundo métodos citados em Kiehl (1985).

Mudas da alface cultivar Babá foram transplantadas no espaçamento de $25 \times 25 \mathrm{~cm}$, no dia 29/8/90 e, após 45 dias no campo, foram colhidas duas plantas/parcela, em quatro repetições, para avaliação da perda de água, em ambiente normal, e cinco plantas/parcela, para avaliação da degradação da clorofila, em câmara fria, também em quatro repetições. Ao final do cultivo, foi determinado o teor de $\mathrm{K}$ disponível nas parcelas, segundo o método de Vettori (1969). O teor de matéria seca (\% peso da matéria fresca da parte aérea) foi determinado por meio da média de quatro plantas/parcela, após secagem em estufa de ventilação forçada, a $60^{\circ} \mathrm{C}$, por 48 horas

A perda de matéria fresca foi avaliada em plantas mantidas em ambiente coberto (temperatura média de $23^{\circ} \mathrm{C}$ e 70\% de UR), e pesadas de 12 em 12 horas após a colheita (HAC), até 84 horas. O valor da parcela foi obtido da média entre duas plantas e os dados expressos em perda de peso acumulada no período, de modo que no momento da colheita a perda foi igual a zero.

O teor de clorofila foi avaliado segundo o método de Withman et al. (1971), no momento da colheita, e aos 3 , 6, 9 e 12 dias após a colheita (DAC), em plantas embaladas individualmente em sacos de polietileno de capacidade para $3,0 \mathrm{~kg}$, e conservadas em câmara fria $\left(4^{\circ} \mathrm{C}\right.$ e $65 \%$ de UR). A degradação da clorofila foi acompanhada nos tratamentos $\mathrm{ORG}_{0} \mathrm{MIN}_{0} ; \mathrm{ORG}_{0} \mathrm{MIN}_{1} ; \mathrm{ORG}_{45,6} \mathrm{MIN}_{0}$; $\mathrm{ORG}_{45,6} \mathrm{MIN}_{1} ; \mathrm{ORG}_{91,2} \mathrm{MIN}_{0} ; \mathrm{ORG}_{91,2} \mathrm{MIN}_{1}$; cada amostra foi tomada na nona folha, contada de fora para dentro, por ser seguramente uma folha não senescente no dia da colheita. Em cada dia de determinação foram analisadas quatro repetições de uma planta cada, com os valores expressos em $\mathrm{mg}$ de clorofila total $/ \mathrm{g}$ de tecido fresco.

A avaliação da perda de matéria fresca e da degradação da clorofila foi realizada no delineamento de parcelas subdivididas, com as fertilizações constituindo as parcelas, e as variáveis HAC e DAC, constituindo as subparcelas.

Os dados obtidos foram submetidos a análise de variância, seguida de regressão, quando pertinente, adotando-se sempre o nível mínimo de $5 \%$ de probabilidade

\section{Resultados e Discussão}

A perda de matéria fresca pelas plantas diminuiu com o incremento das doses de composto orgânico (Figura 1), embora este mesmo incremento proporcionasse maiores teores de água nas plantas (Figura 2). As equações ajustadas encontram-se na Tabela 1. As maiores diferenças entre os tratamentos ocorreram nas primeiras 12 horas (Figura 1), com o que se confirma serem as primeiras horas após a colheita as mais críticas na determinação da perda de água pelas folhas (Ben-Yehoshua \& Aloni, 1974). 


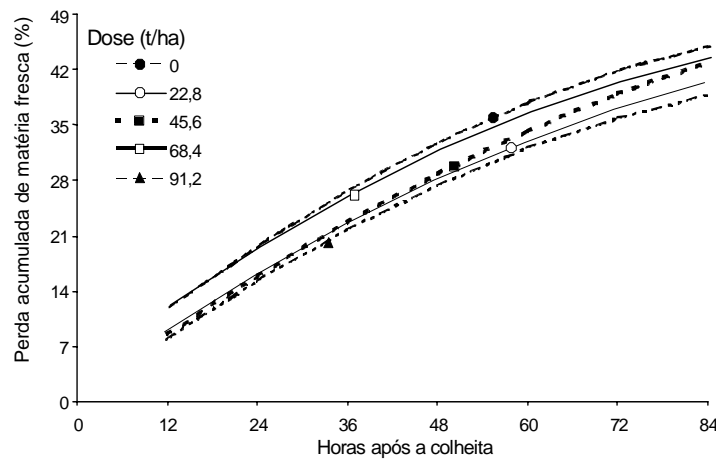

Figura 1. Efeito de doses de composto orgânico sobre a perda de matéria fresca da parte aérea de alfaces Babá mantidas a $23^{\circ} \mathrm{C}$ e $70 \%$ de UR. Médias de dois níveis do fator adubo mineral (sem e com).

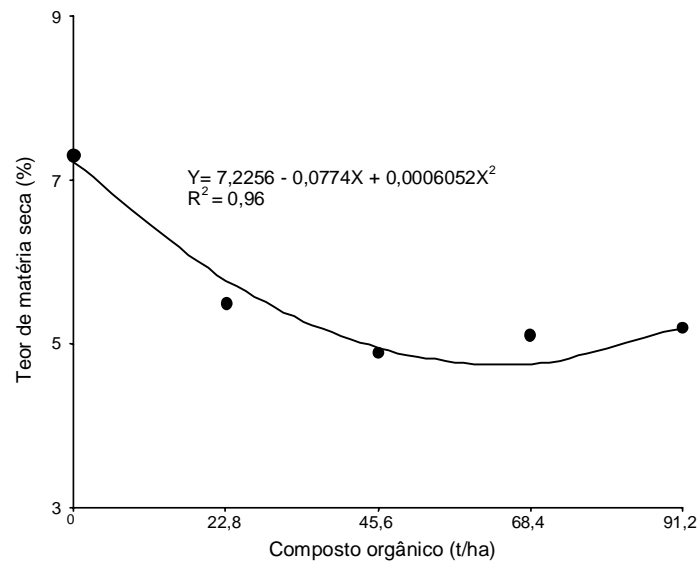

Figura 2. Efeito de doses de composto orgânico sobre o teor de matéria seca (\% do peso da matéria fresca total) da parte aérea de alfaces Babá. Médias de dois níveis do fator adubo mineral (sem e com).
A recuperação da turgidez e a conservação póscolheita de folhosas são incrementadas por altos teores de açúcares no momento da colheita (Yano \& Hayami, 1978a; Stanley, 1989; Amarante, 1991). Contudo, foi verificado que os teores de açúcares solúveis decresceram com o incremento das doses de composto orgânico (Santos et al., 1994), admitindo-se, portanto, a existência de outros mecanismos relacionados aos resultados obtidos. A maior produção de matéria fresca obtida com o incremento das doses de composto orgânico levou a uma maior sobreposição foliar durante o período após a colheita (Santos et al., 1994), acarretando maior contato de parte da superfície foliar com superfície mais úmida, e menor déficit de pressão de vapor nestas condições, ocorrendo uma menor perda de água nas plantas (Wills et al., 1981; Amarante, 1988), quando comparadas às de menor crescimento. Ao lado deste mecanismo, há relato da presença de colênquima mais espessa em plantas fertilizadas organicamente (Schuphan, 1974), aumentando, assim, a resistência à difusão de vapor d'água. Paralelamente, o maior suprimento de K (Figura 3) - elemento que possui papel-chave no controle da resistência estomática à transpiração (Sharma \& Singh, 1990) -, a maior assimilação de $\mathrm{N}$ - proveniente do composto orgâni$\mathrm{co}$, em solutos osmoticamente ativos - e a presença de $\mathrm{NO}_{3}^{-}$vacuolar podem ter conferido às plantas adubadas organicamente maior resistência às tensões hídricas (Machado \& Magnavaca, 1991).

Os teores de clorofila decresceram durante 0 armazenamento somente nas plantas adubadas conjuntamente com composto orgânico e adubo mineral

Tabela 1. Equações de regressão ajustadas para perda de água acumulada ( $\%$ do peso inicial) em plantas de alface, como variável dependente (Y) de horas após a colheita (HAC), em cinco doses de composto orgânico. Médias de dois níveis de adubo mineral (sem e com).

\begin{tabular}{ccc}
\hline $\begin{array}{c}\text { Composto orgânico } \\
\text { (t/ha) }\end{array}$ & Equações & $\mathrm{R}^{2}$ \\
\hline 0,0 & $\mathrm{Y}=2,3522+0,7931^{* *} \mathrm{HAC}-0,3451.10^{-2} * * \mathrm{HAC}^{2}$ & 0,99 \\
22,8 & $\mathrm{Y}=2,9066+0,7454 * * \mathrm{HAC}-0,3202.10^{-2} * * \mathrm{HAC}^{2}$ & 0,99 \\
45,6 & $\mathrm{Y}=0,1759+0,7075^{* *} \mathrm{HAC}-0,2375.10^{-2} * * \mathrm{HAC}^{2}$ & 0,99 \\
68,4 & $\mathrm{Y}=0,8375+0,6957^{*} \mathrm{HAC}-0,2669.10^{-2} * * \mathrm{HAC}^{2}$ & 0,99 \\
91,2 & $\mathrm{Y}=-0,6377+0,7292^{*} \mathrm{HAC}-0,3115 \cdot 10^{-2} * * \mathrm{HAC}^{2}$ & 0,99 \\
\hline
\end{tabular}

** Significativo pelo teste $\mathrm{t}(\mathrm{P}<0,01)$. 
(Figura 4), indicando, assim, efeito aditivo, que acelerou a senescência da alface, uma vez que a degradação da clorofila está intimamente correlacionada ao processo de senescência de folhosas e à perda de suas características comerciais (Silva, 1980; Medina et al., 1982; Lipton, 1987). Este resultado é contrário ao esperado, quando se considera a perda de água pelas plantas em ambiente normal (Figura 1), indicando

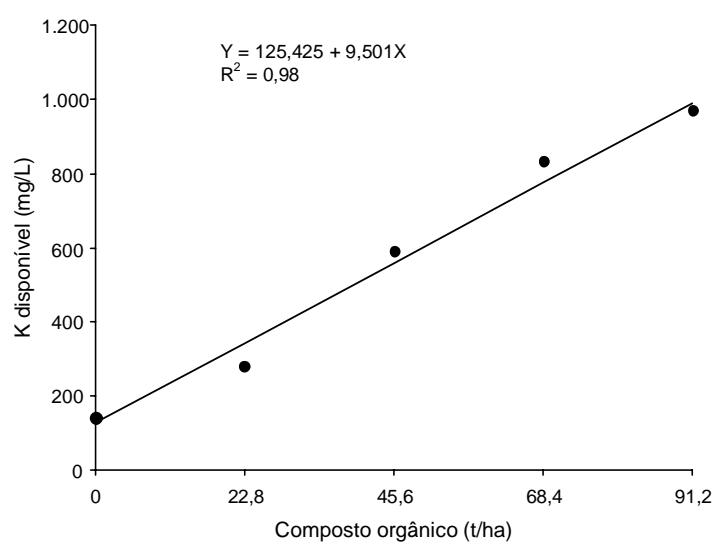

Figura 3. Efeito de doses de composto orgânico sobre o teor de potássio $(\mathrm{K})$ no solo ao final do cultivo. Médias de dois níveis de adubo mineral (sem e com)

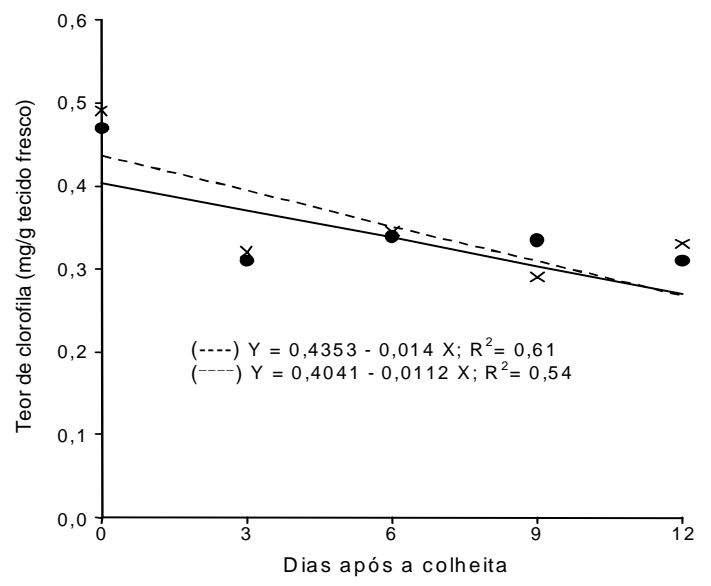

Figura 4. Efeito das doses de 45,6 t/ha (----) e 91,2 t/ha (-) de composto orgânico sobre o teor de clorofila nas folhas de alface 'Babá' mantidas a $40^{\circ} \mathrm{C}$ e $65 \%$ de UR. Médias dos tratamentos que receberam adubação mineral. Valores observados: e $\times$ referentes a 45,6 t/ha e $91,2 \mathrm{t} /$ ha de composto orgânico, respectivamente. que as condições de armazenamento foram eficientes em minimizar esta perda, e atribuindo-se as quedas nos teores de clorofila observados às diferenças qualitativas entre as plantas, em decorrência da aplicação dos diferentes tratamentos. Nos demais tratamentos, o teor de clorofila manteve-se em torno de $0,5 \mathrm{mg} / \mathrm{g}$ de tecido fresco, durante o período de armazenamento

Santos et al. (1994) verificaram que os teores de açúcares solúveis decresceram tanto com o incremento das doses de composto orgânico quanto com o fornecimento de adubo mineral, o que pode reduzir a conservação pós-colheita da alface (Yano \& Hayami, 1978a, 1978b; Nilsson, 1988; Hara, 1989; Amarante, 1991). A queda nos teores de açúcares solúveis está correlacionada positivamente com o fornecimento de altas dosagens de $\mathrm{N}$ solúvel às culturas (Yano \& Hayami, 1978a; Asano, 1984; Hara, 1989), provavelmente ocorrido com a aplicação de altas dosagens de composto orgânico e de adubo mineral, que teriam estabelecido uma correlação negativa entre altos teores de $\mathrm{N}$ nos tecidos e seus teores de açúcares, e sua conservação pós-colheita (Yano \& Hayami, 1978a; Nilsson, 1988).

\section{Conclusões}

1. A aplicação de doses crescentes de composto orgânico proporciona plantas de alface com menor teor de matéria seca, mas promove menor perda de matéria fresca em condições de ambiente natural após a colheita.

2. Na presença de adubo mineral, as doses de 45,6 t/ha e 91,2 t/ha de composto orgânico aceleram a senescência de alfaces mantidas a $4^{\circ} \mathrm{C}$ e $65 \%$ de UR.

\section{Referências}

AMARANTE, C. V. T. do. Efeitos da perda de água sobre a fisiologia pós-colheita de frutos, flores e folhas Viçosa : UFV, 1988. $44 \mathrm{p}$

AMARANTE, C. V. T. do. Relação entre horário de colheita e senescência em folhas de couve (Brassica oleracea L. var. acephala). Viçosa : UFV, 1991. 65 p. Dissertação de Mestrado

ASANO, J. Effect of organic manures on quality of vegetables. Japan Agricultural Research Quarterly, Ibaraki, v. 18, n. 1, p. 31-36, 1984 
BEN-YEHOSHUA, S ; ALONI, B. Effect of water stress on ethylene production of detached leaves of Valencia orange (Citrus sinensis Osbeck). Plant Physiology, Rockville, v. 53, p. 863-865, 1974.

COMISSÃO DE FERTILIDADE DO SOLO DO ESTADO DE MINAS GERAIS (Lavras, MG). Recomendação do uso de corretivos e fertilizantes no Estado de Minas Gerais: $4^{a}$ aproximação. Lavras, 1989. 176 p.

FINGER, L. F.; VIEIRA, G. Controle da perda pós-colheita de água em produtos hortícolas. Viçosa : UFV, 1997. $29 \mathrm{p}$

HARA, T. Effects of nitrogen, phosphorus and potassium in culture solution on the head yield and free sugar composition of cabbage. Japanese Society for Horticultural Science Journal, Tokyo, v. 58, n. 3, p. 595599, 1989

KIEHL, E. J. Fertilizantes orgânicos. Piracicaba : Agronômica Ceres, 1985. 492 p

LIPTON, W. J. Senescence of leafy vegetables. HortScience, Alexandria, v. 22, n. 5, p. 854-859, 1987.

MACHADO, A. T:; MAGNAVACA, R. Estresse ambiental: o milho em perspectiva. Rio de Janeiro : Assessoria em Projetos de Tecnologias Alternativas, $1991.47 \mathrm{p}$

MEDINA, P. V. L.; SILVA, V. F. da; CARDOSO, A. A.; CAMPOS, J. P. de. Perda da qualidade da alface (Lactuca sativa L.) durante o armazenamento. I. Relações entre mudanças metabólicas. Revista Ceres, Viçosa, v. 29, n. 163, p. $259-267,1982$

NILSSON, T. Growth and carbohydrate composition of white cabbage for long-term storage. I. Effects of late $\mathrm{N}$-fertilization and time of harvest. Journal of Horticultural Science, Ashford, v. 63, n. 3, p. 419-429, 1988

SANTOS, I. C. dos; VICENTE, V. W. D.; MIRANDA, G. V. Comportamento de dez cultivares de alface adubadas com composto de lixo urbano. Pesquisa Agropecuária Brasileira, Brasília, v. 33, n. 2, p. 157-161, fev. 1998.

SANTOS, R. H. S.; CASALI, V. W. D.; CONDÉ, A. R.; MIRANDA, L. C. G. de. Qualidade de alface cultivada com composto orgânico. Horticultura Brasileira, Brasília, v. 12, n. 1, p. 29-32, 1994.
SCHUPHAN, W. Nutritional value of crops as influenced by organic and inorganic fertilizers treatments. Qualitas Plantarum, Dordrecht, v. 13, n. 4, p. 333-358, 1974.

SHARMA, C. P.; SINGH, S. Sodium helps overcome potassium deficiency effects on water relations of cauliflower. HortScience, Alexandria, v. 25, n. 4, p. 458459,1990

SILVA, V. F. Modificações bioquímicas e aparentes do processo de senescência em alface (Lactuca sativa $\mathbf{L}$.) durante o armazenamento. Viçosa : UFV, 1980. 39 p. Dissertação de Mestrado.

STANLEY, R. The influence of temperature and packaging material on the post harvest quality of iceberg lettuce. Acta Horticulturae, Leuven, v. 2, n. 244, p. 171-177, 1989.

VETTORI, I. Métodos de análise de solo. Rio de Janeiro : Ministério da Agricultura, 1969. 24 p.

WILlS, R. H. H.; LEE, T. H.; GRAHAM, D.; McGLASSON, W. B.; HALL, E. G. Postharvest. Westport : AVI, 1981. $163 \mathrm{p}$.

WITHMAN, F. H; BLAYDES, D. F.; DEWIN, R. Experiments in plant physiology. New York : Von Nostrand, 1971. $175 \mathrm{p}$.

YANO, M.; HAYAMI, A. Studies on the improvement of storage ability in the head vegetables. I. The relationship between cultivars, maturity rates and fertilizer and storage ability of lettuce and cabbage. Tsu : National Research Institute of Vegetable, Ornamental Plants and Tea, 1978a. p. 77-88. (Yasai Shikenjo Hokoku Station. Bulletin of Vegetables and Ornamental Crops Research. Series, A4)

YANO, M.; HAYAMI, A. Studies on the improvement of storage ability in the head vegetables. II. The relationship between the post harvest physiological characteristics and storage ability of lettuce. Tsu : National Research Institute of Vegetable, Ornamental Plants and Tea, 1978b. p. 89101. (Yasai Shikenjo Hokoku Station. Bulletin of Vegetables and Ornamental Crops Research. Series, A4). 